\title{
miR-487b-3p impairs osteoblastogenesis by targeting Notch-regulated ankyrin-repeat protein (Nrarp)
}

\author{
Aijaz A John, Ravi Prakash and Divya Singh \\ Division of Endocrinology and Centre for Research in Anabolic Skeletal Targets in Health and IIIness (ASTHI), CSIR-Central Drug Research Institute, \\ Lucknow, India
}

Correspondence should be addressed to D Singh: divya_singh@cdri.res.in

CDRI communication number 9836

\begin{abstract}
miRNAs have appeared as critical controllers of gene expression at post-transcriptional level either by degrading RNA transcripts or repressing translation. It is evident from the ever-growing scientific literature that miRNAs play a significant role in osteoblast commitment and differentiation. Here, we report that overexpression of miR-487b-3p leads to inhibition of osteoblastic differentiation. Using in silico approaches, Nrarp was found to be the direct target of miR-487b-3p, which was further validated by luciferase 3' UTR reporter assay. Nrarp inhibits Notch-1 signaling and promotes Wnt signaling by stabilization of LEF-1. Role of miR-487b-3p in regulating canonical Wnt and Notch signaling was determined by western blotting. Protein levels of Nrarp, RUNX-2, Lef1 and $\beta$ catenin were reduced in osteoblasts cells transfected with miR-487b-3p, whereas protein levels of Notch1, Hes1 and P- $\beta$ catenin were upregulated when osteoblast cells were transfected with miR-487b-3p. These outcomes were reversed after treating cells with anti-miR-487b-3p. Further silencing of miR-487b-3p in neonatal Balb/c mice attenuated all the inhibitory actions of miR-487b-3p on osteoblast differentiation. Importantly, in vivo action of anti-miR-487b-3p to ovariectomized osteopenic BALB/c mice steered to significant enhancement in trabecular bone microarchitecture. Furthermore, the bio-mechanical properties of isolated femurs were enhanced in anti-miR-487b-3ptreated mice. Overall, miR-487b-3p negatively regulates osteogenesis by suppressing Nrarp expression, which in turn, suppresses Runx-2 and Wnt signaling, both of which play a pivotal action in osteoblast differentiation.
\end{abstract}

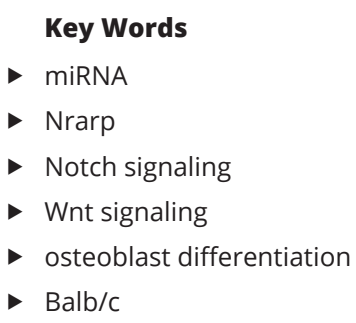

Key Words

miRNA

- Nrarp

- Notch signaling

- Wnt signaling

- Balb/c
Journal of Endocrinology (2019) 241, 249-263

\section{Introduction}

A tight regulation of gene activation and repression is required for bone development and homeostasis. Pathways like Wnt signaling and bone morphogenetic proteins (BMPs) activate transcriptional programs of mesenchymal stem cells (MSCs) that are required for the commitment toward the osteoblast lineage (Chen et al. 2016). Furthermore, it has been revealed by several in vitro studies that osteoblastic and osteoclastic differentiation has been related with Notch signaling (Regan \& Long 2013). Loss and gain of Notch 1 function studies revealed 
that Notch signaling acts in a temporal and contextdependent mode in osteoblast differentiation (Bae et al. 2012). Studies have revealed that gain of Notch 1 function within the initial osteoprogenitor compartment shows inhibition of osteoblastic commitment and low bone mass (Regan \& Long 2013, Zanotti \& Canalis 2016). On the other hand, gain of function in committed osteoblastic cells encourages proliferation of immature osteoblasts, whereas inhibiting terminal differentiation of osteoblasts (Zanotti \& Canalis 2016).

Notch signaling not only plays an important role in cell-fate determination and cell differentiation but also plays an important role in cell survival and cell death processes (Dontu et al. 2004). The pathway consists of four receptors i.e. Notch1, Notch2, Notch3 and Notch4 and several ligands which include Jagged1, Jagged2, Delta-like ligand 1, Delta-like ligand 3 and Delta-like ligand 4 in mammals (Furukawa et al. 2016). The pathway is initially activated by occupation of the representative Notch receptor. Subsequently, transmembrane domain of the Notch receptor is cleaved by a $\gamma$-secretase complex which results in the release of the intracellular domain of the Notch receptor (NICD) (Furukawa et al. 2016). The released NICD then moves to the nucleus and initiates transcription of Notch downstream targets (Regan \& Long 2013, Furukawa et al. 2016). Activation or overexpression of Notch suppresses osteoblastic differentiation mainly through inhibition of Wnt/ $\beta$-catenin signaling, which is also a critical regulator of osteoblastogenesis (Yavropoulou \& Yovos 2014). Zanotti et al. reported that in NICD overexpressing mice presented with a decrease in bone volume which was secondary to a reduction in trabecular number (Zanotti et al. 2008). NICD overexpression also led to decrease in osteoblast and osteoclast numbers (Zanotti et al. 2008). They reported that Notch signaling in osteoblasts results in osteopenia and impaired osteoblastogenesis by obstructing the $\mathrm{Wnt} / \beta$-catenin pathway (Zanotti et al. 2008). Deregowski et al. also showed that overexpression of Notch 1 inhibited osteoblastogenesis by suppressing Wnt/ $\beta$-catenin (Deregowski et al. 2006).

Recent studies have revealed that miRNAs play a crucial role in osteoblast functions and development (Kureel et al. 2014, 2017, 2018, John et al. 2018). These miRNAs not only play significant roles in the development, differentiation and function of different cell types but also play an important role in the pathogenesis of various human diseases (Kureel et al. 2017). It has been found that the highly conserved Notch pathway functions recurrently to regulate many fundamental cellular processes in animal development (Poulton et al. 2011). For instance, in Drosophila there have been many reports revealing that there is a direct association between miRNA regulation and Notch signaling. Several downstream target genes of Notch signaling including the $\mathrm{E}(\mathrm{spl})$ and Bearded (Brd) family of transcription factors have been identified as miRNA targets in vivo (Lai et al. 2005). In case of bone, microRNA-199b-5p is associated in the Notch signaling pathway in osteosarcoma (Won et al. 2013). Bae et al. have established the role of miRNA-34c in regulating Notch signaling during bone development (Bae et al. 2012).

Our previous studies identified several miRNAs to be downregulated by medicarpin, an osteoprotective agent (Kureel et al. 2014, 2017, 2018, John et al. 2018). One of the miRNAs identified was miR-487b-3p which was more than 6.5-fold suppressed in response to medicarpin. However, role of miR-487b-3p is not known in the regulation of osteoblast functions. In this study, we establish that miR-487b-3p negatively controls osteogenesis by obstructing Notch-regulated ankyrin-repeat protein (Nrarp) which blocks Notch signaling and promotes bone formation.

\section{Materials and methods}

\section{Chemicals and reagents}

All cell culture media including fetal bovine serum (FBS), antibiotic solution, $\alpha$-MEM, sodium pyruvate and nonessential amino acid were acquired from Sigma. miRNA isolation kit (mirVana), miR-487b-3p, anti-miR-487b-3p, negative control, in vivo ready miRNA, TaqMan miRNA reverse transcriptase kit, TaqMan universal master mix, Lipofectamine RNAimax and Opti-MEM were procured from Ambion (Applied Biosystems). Antibodies were obtained from Abcam (Cambridge Science Park, Cambridge, UK), CST, Santa Cruz Biotechnology and R\&D Systems. pEZX-MT01 vector in which 3' UTR of Nrarp was cloned ( $3^{\prime}$ UTR Nrarp clone) and empty pEZX-MT01 vector (control clone) was purchased from Genecopia (Rockville, MD USA). Dual-glow luciferase assay system was purchased from Promega. CTX and P1NP ELISA kits were procured from Elabscience (Elabscience Biotechnology Inc., Wuhan, Hubei).

\section{Mice osteoblast cell culture}

To initiate osteoblast culture, mouse pups aging 1-2 days old were used to isolate calvarial osteoblasts. The culture was carried out as per previously published protocol 
of five sequential digestions (Kureel et al. 2014, 2017, 2018). In brief, calvariae from the skull were surgically detached and were chopped into small fragments. The chopped calvarial fragments were exposed to five sequential enzymatic digestions. To release cells from calvarial fragments enzymatic digestions were carried out for $10-15 \mathrm{~min}$ at $37^{\circ} \mathrm{C}$ in $0.1 \%$ collagenase $\mathrm{P}$ and $0.1 \%$ dispase. The cells from first digestion were discarded as it contains heterogeneous population of cells. Fetal calf serum $3-4 \mathrm{~mL}$ was used to collect cells from the rest digestions i.e. second to fifth enzymatic digestions. The cells collected from second to fifth enzymatic digestions were combined and centrifuged at $1800-2225 \boldsymbol{g}$ for $5 \mathrm{~min}$. The cells were resuspended and plated in T-25- $\mathrm{cm}^{2}$ flasks in complete growth medium i.e. $\alpha$-minimal essential medium ( $\alpha$-MEM) containing 10\% FCS and 1\% penicillin/ streptomycin. For the incubation of cells, the T-25- $\mathrm{cm}^{2}$ flasks were kept in a humidified incubator at $37^{\circ} \mathrm{C}$ and $5 \%$ $\mathrm{CO}_{2}$. Media of the cells was changed after every $24 \mathrm{~h}$. The osteoblast cells were trypsinized after reaching 70-80\% confluence and then re-seeded in cell culture plates in complete growth medium for further experiments.

\section{miRNA microarray and data analysis}

Microarray procedure and data analysis was executed at Genotypic Pvt Ltd. in a protocol using Agilent miRNA Hybridization kit as described earlier (Kureel et al. 2014, 2017, 2018)

\section{qRT-PCR analysis for miRNA}

To validate the microarray data, qRT-PCR analysis for miR-487b-3p was carried out using RNA isolation in combination with TaqMan miRNA reverse transcription kit and a TaqMan microRNA assay kit (Applied Biosystems) for quantification according to earlier available protocol (Kureel et al. 2014, 2017, 2018). In brief, total RNA was isolated from mice calvarial osteoblast cells (MCOs) by means of mirVana miRNA isolation kit (Ambion) using manufacturer's protocol and cDNA was constructed through reverse transcription and was used as template for real-time PCR. After cDNA synthesis quantitative real-time PCR (qRT-PCR) was done by means of TaqMan miRNA assay kit (Applied Biosystems) according to manufacturer's protocol.

Initially, the reaction mixtures $(15 \mu \mathrm{L})$ were incubated at $95^{\circ} \mathrm{C}$ for $10 \mathrm{~min}$. After initial incubation of $10 \mathrm{~min}$ reaction mixtures were followed by 40 cycles of $95^{\circ} \mathrm{C}$ for $15 \mathrm{~s}$ and at $60^{\circ} \mathrm{C}$ for $1 \mathrm{~min}$ using the Step One Plus Real-Time
PCR system (Applied Biosystems). Triplicate reactions were carried out to achieve the mean Ct values of each sample. The $\log _{2} \mid 2-$ DCt $\mid$ method was used to check the relative expression level of the concerned miRNA. In this method, DCt was calculated as the subtraction of the Ct value of the target miRNA from the Ct value of internal control U6.

\section{Medicarpin treatment in Balb/c mice}

BALB/c mouse model was selected to perform this study. The experiments were executed in accordance with current regulation of animal experiments i.e. according to the rules laid down by Institutional Animal Ethical Committee (IAEC) at Central Drug Research Institute (CDRI), Lucknow. These animal ethical rules and regulations were taken from the National Laboratory Animal Centre, CSIR-CDRI. Animals were divided in two groups and each group carried eight animals. One group of Balb/C mice were used as control and the other was treated with medicarpin. Control group of animals were treated with gum acacia mixed in distilled water and the other group of Balb/C mice were treated with medicarpin $(10.0 \mathrm{mg} / \mathrm{kg} /$ day $)$ orally for 1 month. At the end of 1 month, animals were killed and long bones (femur and tibia) were collected for RNA isolation. Bones were crushed in liquid nitrogen for RNA isolation using mirVana miRNA isolation kit purchased from Ambion (Applied Biosystems).

\section{Transfection assay}

Transfections of miR-487b-3p, anti-miR-487b-3p and miC (negative control) were carried into mouse osteoblasts isolated from neonatal BALB/c mice pups. Lipofectamine RNAi MAX reagent was used as a transfection media in Opti-MEMI Reduced Serum Medium (Invitrogen) as per manufacturer's procedure. One day ahead of transfection mouse calvarial osteoblasts were seeded in cell culture plates in complete growth media ( $\alpha$-MEM comprising $10 \%$ FCS and $1 \%$ penicillin/streptomycin). After attaining $50-70 \%$ confluence, these were transfected with miR-487b-3p, anti-miR-487b-3p and negative control (miC) (Ambion) at $50 \mathrm{nM}$ concentration using Lipofectamine RNAi MAX. The transfection was carried for $6 \mathrm{~h}$ with reduced serum and antibiotic-free Opti-MEM. After $6 \mathrm{~h}$, medium was changed and the cells were allowed to grow in differentiation medium containing $50 \mu \mathrm{g} / \mathrm{ml}$ ascorbic acid, 10-mM $\beta$-glycerophosphate, and 10\% FCS (complete growth medium). For further analysis, cells were collected after $48 \mathrm{~h}$ of miRNA transfection. 


\section{Alkaline phosphatase activity}

ALP activity was measured as per earlier available protocols (Kureel et al. 2014, 2017, 2018). Briefly, mouse calvarial osteoblasts at $70-80 \%$ confluence were trypsinized and re-seeded in 96-well plate (2000 cells/ well). This was followed by transfection of cells with negative control (miC), miR-487b-3p (mimic) and anti-miR-487b-3p (inhibitor) at 50nM concentration. The incubation of the transfected cells was carried out for $48 \mathrm{~h}$ in the differentiation medium i.e. media comprising of $10 \mathrm{mM} \beta$-glycerophosphate, $50-\mu \mathrm{g} / \mathrm{mL}$ ascorbic acid and $10 \%$ FCS. After the completion of incubation period, total ALP activity was calculated using nitrophenylphosphate (PNPP) as substrate and absorbance was taken at $405 \mathrm{~nm}$.

\section{Measurement of mineralized matrix formation}

For mineralization assay, mouse calvarial osteoblasts (MCOs) were isolated and cultured according to earlier available protocols from our laboratory (Kureel et al. 2014, $2017,2018)$. In brief, after trypsinization cells were re-seeded $\left(2.5 \times 10^{3}\right.$ cells/well in 12-well plate) in differentiation media with $10 \%$ FCS, cells were transfected with miC (negative control), miR-487b-3p (mimic) and anti-miR-487b-3p (inhibitor) using Lipofectamine RNAi MAX as a transfection agent at $50 \mathrm{nM}$ concentration. Following transfection, cells were incubated for 21 days at $37^{\circ} \mathrm{C}$. The media of the plate was changed every $48 \mathrm{~h}$. At the end of 21 days, attached cells were washed gently with PBS. After washing with PBS attached cells were fixed in formaldehyde for $20 \mathrm{~min}$ at room temperature. The fixed cells in each well were stained with $40 \mathrm{mM}$ alizarin red for $30 \mathrm{~min}$. Stained calcium nodules were observed and were photographed under a light microscope. Quantification of calcium nodules was then carried out using standard protocol.

\section{miRNA target site prediction}

The potential targets were screened for miR-487b-3p using TargetScan (Release 7.1: 2016) (http://www.targetscan.org) and mirdb (http://www.mirdb.org). This computational target prediction was primarily based on the potential pairing of the miRNA seed sequence to the complementary site in the 3'UTR of a target mRNA according to specific base-pairing rules (John et al. 2004, Grimson et al. 2007).

\section{Luciferase reporter assay}

Luciferase reporter assay was carried out according to the earlier published protocols from our laboratory
(Kureel et al. 2014, 2017, 2018). Primary osteoblast cells at $85-90 \%$ confluence were transfected with $200 \mathrm{ng}$ pEZX-MT01 vector in which 3'UTR of Nrarp was cloned with Lipofectamine RNAi MAX for $6 \mathrm{~h}$ in reduced serum and antibiotic-free Opti-MEM. The clone purchased from Genecopia enclosed firefly luciferase as the reporter gene controlled by SV40 promoter gene and Renilla luciferase as the tracking gene controlled by CMV promoter. Cells were co-transfected with the mimic miR-487b-3p or a negative control (miR control) (Applied Biosystems) at concentrations of $50 \mathrm{nM}$. Firefly and Renilla luciferase expressions were determined in cell lysates by means of a Dual-Luciferase Reporter Assay System acquired from Promega on a FLUOstar galaxy (BMG Lab Technologies, Melville, NY, USA). Renilla luciferase activity was used for normalization and as an internal control for transfection efficiency. Relative luciferase activity (firefly/renilla) was expressed in relative luminescence units and plotted.

\section{qRT-PCR analysis for osteogenic genes}

For gene expression studies, cells were transfected with miC (negative control), miRNA-487b-3p (mimic) and antimiR-487b-3p (inhibitor) using Lipofectamine RNAi MAX as a transfection agent. After $48 \mathrm{~h}$ of incubation, whole RNA was extracted from the cultured cells using TRIzol (Life Technologies). cDNA was synthesized by using $2 \mu \mathrm{g}$ total RNA with the Revert Aid H Minus first-strand cDNA synthesis kit (Fermentas, Hanover, MD, USA). Syber green chemistry was used for quantitative assessment of mRNAs for ALP, Nrarp, RUNX-2, type I collagen, OCN, BMP-2, Hes1, $\beta$ - Catenin ( $\beta$-cat) and a housekeeping gene GAPDH following a previously published protocol (Kureel et al. 2014, 2017, 2018). The design of sense and antisense oligonucleotide primers was based on published cDNA sequences using the Universal Probe Library (Roche Diagnostics). Primer sequences are given in Table 1. For real-time PCR, Light Cycler 480 (Roche Diagnostics) was used for cDNA amplification. The temperature summary of the qPCR reaction was $95^{\circ} \mathrm{C}$ for $5 \mathrm{~min}, 40$ cycles of denaturation at $94^{\circ} \mathrm{C}$ for $2 \mathrm{~min}$ followed by annealing at $62^{\circ} \mathrm{C}$ for $30 \mathrm{~s}$, extension at $72^{\circ} \mathrm{C}$ for $30 \mathrm{~s}$. For the normalization of differences in RNA degradation, RNA isolation and the efficiencies of the reverse transcription, GAPDH was utilized to normalize contrasts.

\section{Western blot analysis}

For western blot analysis, MCO cells were seeded in six-well plates in complete growth media ( $\alpha$-minimal 
Table 1 Primer sequences.

\begin{tabular}{l}
\hline Gene \\
\hline Nrarp forward: \\
Nrarp reverse: \\
Runx-2 forward: \\
Runx-2 reverse: \\
Bmp-2 forward: \\
Bmp-2 reverse: \\
Ocn forward: \\
Ocn reverse: \\
$\beta$-Catenin forward: \\
B-Catenin reverse: \\
Hes-1 forward: \\
Hes-1 reverse: \\
Type1 collagen forward: \\
Type1 collagen reverse: \\
Alp forward: \\
Alp reverse: \\
Gapdh forward: \\
Gapdh reverse:
\end{tabular}

\section{In vivo study (experimental design and related methods)}

All studies were carried out on female Balb/c mice in accordance with current legislation on animal experiments (IAEC) at CDRI. The experiments were executed in accordance with current regulation of animal experiments, i.e. according to the rules laid down by CSIRCentral Drug Research Institute, Institutional Animal Ethical Committee (IAEC) Registration No.: 34/GO/Re-SL/ Bi-S/99 CPCSEA Renew date 13.04.2017 under project approval code no. IAEC/2017/F-290 dated 31.10.2017.

Balb/c mice (8-10 weeks old) weighing $25 \pm 3 \mathrm{~g}$ were randomly divided in four groups (sham, negative control, mimic and inhibitor). Three groups (negative control, mimic and inhibitor) underwent ovariectomy, whereas the fourth group was sham operated. The three groups, i.e., negative control group, mimic group and the inhibitor group were given one injection/week subcutaneously for 3 weeks with $\mathrm{miC}$ (negative control), miR-487b-3p (mimic) and anti-miR-487b-3p (inhibitor) (Ambion). Solutions of miC, miR-487b-3p and of anti-miR-487b-3p were each diluted with in vivo-jetPEI solution (PolyPlus Transfection, Illkirch, France) comprising 10\% (w/v) glucose (Polyplus Transfection reagent) at an N/P ratio of 8 , following the references of the manufacturer (Polyplus Transfection). All the solutions carried a $5 \mu \mathrm{g}$ oligonucleotide per dose. Prior to injection all the solutions were mixed by vortexing for $10 \mathrm{~s}$ and incubated for at least $15 \mathrm{~min}$ at $37^{\circ} \mathrm{C}$. It was made sure that each female Balb/c mouse received $100 \mu \mathrm{L}$ of oligonucleotide mixture via subcutaneous injection behind the head. The $100 \mu \mathrm{L}$ of oligonucleotide mixture contained $5 \mu \mathrm{g}$ of oligonucleotide with $\mathrm{N} / \mathrm{P}$ ratio of 8 $(0.16 \mu \mathrm{L}$ of in vivo-jetPEI per $\mu \mathrm{g}$ of oligonucleotide). At the completion of 4 th week, mice were killed and serum samples were collected for ELISA. Femur bones along with tibia and fibula were collected for analysis of trabecular microarchitecture using $\mu \mathrm{CT}$.

\section{Micro-computed tomography $(\mu \mathrm{CT})$ analysis}

Micro-computed tomographic $(\mu \mathrm{CT})$ determination of excised bones from various Balb/c groups were carried out using the SkyScan $1076 \mu \mathrm{CT}$ scanner (Sky Scan, Aartselaar, Antwerp, Belgium) as described in earlier available protocols (Kureel et al. 2014, 2017, 2018). Briefly, femora and tibiae were cleaned of soft tissue, and fixed before storage in $70 \%$ isopropanol. The bones were subjected to X-ray source of $70 \mathrm{kV}, 100 \mathrm{~mA}$ with a nominal resolution (pixel size) of $18 \mathrm{~mm}$ for scanning. 
A hundred projections were acquired over an angular range of $180^{\circ}$. This was followed by reconstruction of image slices using a modified Feldkamp algorithm using the Sky Scan Nrecon software, which aided in network-distributed reconstruction. This network-distributed reconstruction was performed on four personal computers running concurrently. Various trabecular bone parameters, i.e., Tb. Sp., trabecular separation; BV/TV., bone volume/tissue volume ratio; Tb.N., trabecular number; Tb. Pf., Trabecular pattern factor, Tb.Th., trabecular thickness; and Conn. D., Connectivity density were calculated.

\section{Bone strength testing}

To check the bio-mechanical properties of bones, mice femurs in each group were subjected to three-point bending by means of a bone strength tester model TK 252C (Muromachi Kikai Co. Ltd., Tokyo, Japan). Each hydrated mice femur was horizontally positioned on the supports. The distance between two supports was kept constant at $1 \mathrm{~cm}$. The load-displacement curves were used to calculate ultimate load $(\mathrm{N})$, energy to fracture $(\mathrm{N}-\mathrm{mm})$, stiffness $(\mathrm{N} / \mathrm{mm})$.

\section{Estimation of bone turnover markers}

Bone turnover markersi.e. type 1 cross-linked C-telopeptide (CTX) and N-terminal type 1 procollagen (P1NP) was measured by commercially available ELISA kits. These ELISA kits were acquired from Elabscience (Elabscience Biotechnology Inc., Donghu Hi-Tec Development area, Wuhan, Hubei). The serum levels of CTX and P1NP were determined by ELISA following the manufacturer's protocols.

\section{Hematoxylin and eosin staining of trabecular bone}

Hematoxylin and eosin staining of trabecular bone was carried out according to the previously published protocol from our laboratory (John et al. 2018). Disarticulation of forelimb was carried out from knee joint to remove femurs. After that skin, muscles and superficial layers of soft tissues were removed. Decalcification of the femurs in various groups i.e. sham group, $\mathrm{miC}$ group (negative control), miR-487b-3p group (mimic) and anti-miR-487b-3p group (inhibitor) were carried out using decalcifying buffer (decalcifying solution lite, Sigma-Aldrich) for at least $72 \mathrm{~h}$. After decalcification epiphyseal portion of the femur was cut from the bone and processed in 70\% isopropanol. This was followed by embedding the tissue in paraffin for sectioning. At the end of sectioning the sections were stained with hematoxylin and eosin (HE) Sigma-Aldrich.

\section{Anti-miR-487b-3p administration in neonatal Balb/c pups}

Transfection of $\mathrm{miC} / \mathrm{mimic} /$ inhibitor in neonatal pups was performed according to the previously published protocols from our laboratory (John et al. 2018). Balb/c pups (1-2 days old) weighing 2-3g were indiscriminately separated in three groups (negative control, mimic and inhibitor). The three groups that is negative control, mimic and inhibitor were transfected with $\mathrm{miC}$, miR-487b-3p and anti-miR-487b-3p respectively using vivo-jetPEI solution (Polyplus Transfection, Illkirch, France). Each group received $0.45 \mu \mathrm{g}$ oligonucleotide subcutaneously in a single injection of $30 \mu \mathrm{L}$. Solutions of each oligonucleotides i.e. miC, miR-487b-3p and antimiR-487b-3p were formulated in in vivo-jetPEI solution comprising 10\% (w/v) glucose (Polyplus Transfection reagent) at an N/P ratio of 6 , following the references of the manufacturer (Polyplus Transfection). This was followed by subcutaneous injection $(30 \mu \mathrm{L})$ of oligonucleotide solution comprising $0.45 \mu \mathrm{g}$ of oligonucleotide with $\mathrm{N} / \mathrm{P}$ ratio of 6 , i.e., $0.12 \mu \mathrm{L}$ of in vivo-jetPEI per $\mu \mathrm{g}$ of oligonucleotide) near the head region (usually behind the head) in neonatal Balb/c pups. At the end of $48 \mathrm{~h}$, animals were killed and calvaria were harvested for RNA isolation.

\section{Statistical analysis}

Data are communicated as mean \pm s.E.M. For experiments with two treatments Student's $t$-test was used to study statistical significance, whereas one-way ANOVA followed by Newman-Keuls test of significance was carried out for experiments with multiple treatments using Graph Pad Prism, version 3.02 software. Although all experiments were repeated thrice but representative experiments are shown in the manuscript.

\section{Results}

\section{miR-487b-3p negatively regulates osteoblast} differentiation

It was observed from our previous study conducted by Kureel et al. (2014) that many miRNA candidates were differentially regulated in medicarpin-stimulated osteoblast cultures. From the microarray data, it was 
revealed that most of the differentially expressed miRNAs were downregulated by medicarpin treatment, whereas only a few of them were upregulated (Kureel et al. 2014). One of the fascinating candidates was miR-487b-3p, which showed 6.5-fold downregulation in medicarpin-treated mouse calvarial osteoblast cells (MCO) compared to the control (Fig. 1A). This data were authenticated by qRT-PCR analysis which also confirmed that expression level of 487b-3p was significantly downregulated in medicarpin and BMP-2-treated osteoblast cells respectively at $24 \mathrm{~h}$ and $48 \mathrm{~h}$ (Fig. 1B).

In addition, we determined miR-487b-3p expression levels in long bones (femur and tibia) extracted from sham, and mice treated with medicarpin $(10 \mathrm{mg} / \mathrm{kg}$ for 1 month). It was observed that miR-487b-3p expression in Med-treated group was significantly reduced compared to sham (Fig. 1C). Thus, our microarray data were reproducible not only at in vitro level but also at in vivo level. After initial validation assays the next question was to establish the role of miR-487b-3p on osteoblast functions.
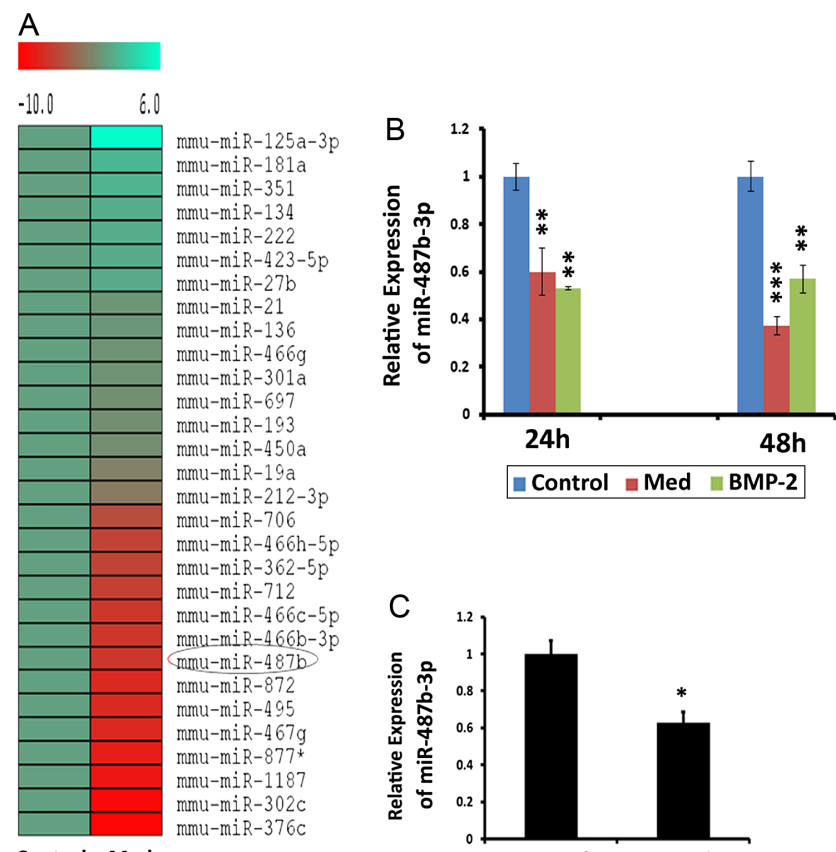

Control Med mmu-miR-376c

\section{Figure 1}

Identification of miRNAs with differential expression during medicarpininduced osteoblast differentiation. (A) miRNA array expression profiling. Red denotes low expression and aqua denotes high expression relative to the median and only representative miRNAs that were significantly downregulated and up regulated are shown. (B) Fold decrease in miR-487b-3p expression during medicarpin and BMP-2-induced murine osteoblast differentiation at $24 \mathrm{~h}$ and $48 \mathrm{~h}$ respectively. (C) miR-487b-3p expression levels in long bones isolated from sham, and mice treated with Med. All values represent means \pm S.E. $(n=6) . * P<0.05$, ** $P<0.01$, $\star * \star P<0.001$ compared with the control. A full colour version of this figure is available at https://doi.org/10.1530/JOE-19-0015.
To find out the role of miR-487b-3p on osteoblast differentiation alkaline phosphatase activity (ALP) was measured spectrophotometrically after $48 \mathrm{~h}$. Alkaline phosphatase activity (ALP) is one of the main osteoblast differentiation marker. Amid this test, the osteoblast cells were transfected with $\mathrm{miC}$ (negative control), miR-487b-3p (mimic) and anti-miR-487b-3p (inhibitor) and prompted to differentiation. It was revealed that compared with cells transfected with miR-C, ALP activity was significantly downregulated in miR-487b-3p-transfected cells. Opposite effects were observed in anti-miR-487b-3ptransfected cells (Fig. 2A). Similar results were perceived at transcriptional levels of ALP (Fig. 2B). Effect of miR-487b-3p was also perceived on mineral nodule formation (an osteogenic marker). Mineral nodule formation was assessed by alizarin staining in 21-day culture, revealing that overexpression of miR-487b-3p resulted in significant decrease in mineral nodule formation (Fig. 2C and D). Reverse results were observed in anti-miR-487b-3p-transfected cells. Additionally, expression of miR-487b-3p also decreased during the course of osteoblast differentiation (Fig. 2E).

Osteoblast development and differentiation is controlled by numerous transcription and growth factors like BMP-2, Runx-2 and matrix proteins like type I collagen (Jensen et al. 2010). The influence of miR-487b-3p on these osteogenic factors was carried out by overexpression of miR-487b-3p and inhibition of miR-487b-3p. It was observed that transfection of miR-487b-3p repressed the expression levels of osteogenic gene markers like type I col, OCN, Runx-2 and BMP2 (Fig. 2F, G, H and I). Cells transfected with anti-miR-487b-3p transfected cells gave opposite effects. Protein levels of type 1 collagen was also repressed in miR-487b-3p-transfected cells while in cells transfected with anti-miR gave opposite results (Fig. 2J and K). Similarly, the effect of miR-487b-3p was observed in human osteoblast cells (hoB) procured from European Collection of Authenticated Cell Cultures (ECACC). During the study it was revealed that miR-487b-3p overexpression in hOB (human osteoblasts) leads to decreased ALP activity (Supplementary Fig. 1A, see section on supplementary data given at the end of this article) and transcript levels of osteogenic markers like RUNX2, type I col and BMP-2 (Supplementary Fig. 1B, C and D). Opposite effects were observed in anti-miR-487b-3p-transfected cells.

\section{miR-487b-3p targets Nrarp with high affinity}

Next stage was to predict the target gene for miR-487b-3p by which it controls osteoblast function. 


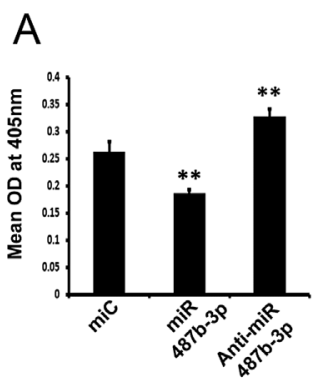

B
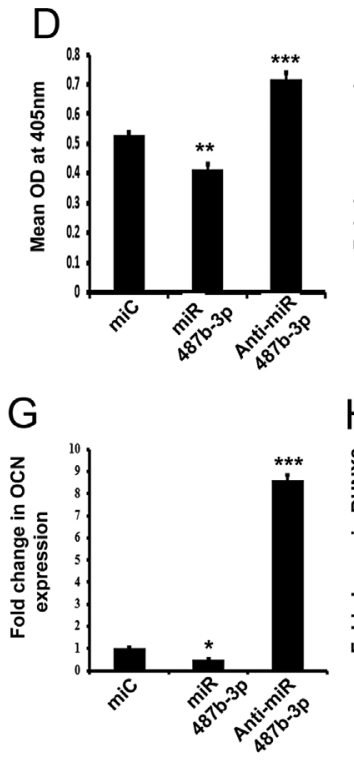

$\mathrm{H}$

E

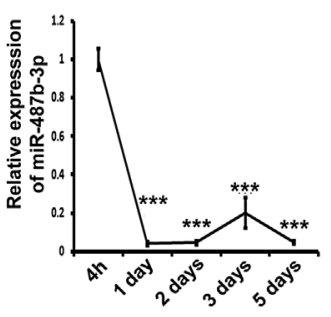

$\mathrm{F}$
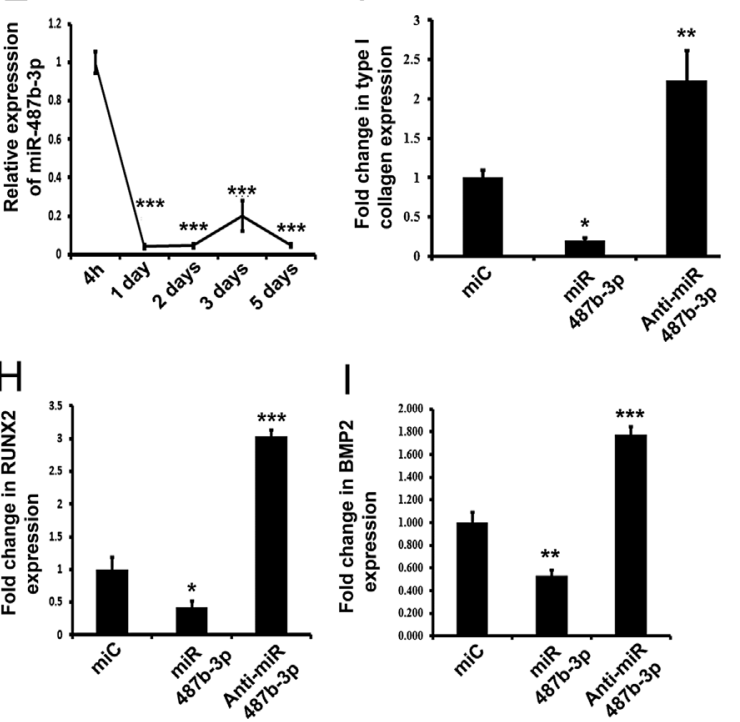

$\mathrm{J}$
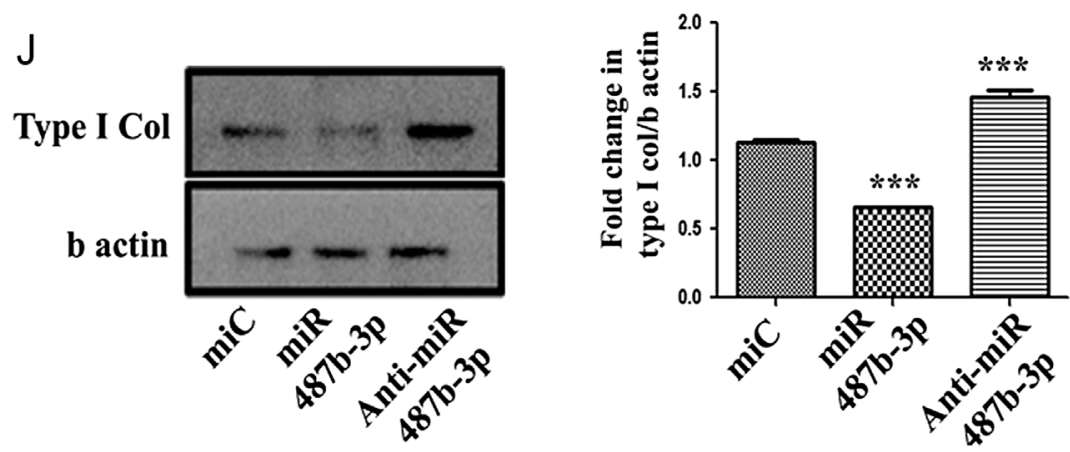

Figure 2

miR-487b-3p regulates osteoblast differentiation. (A) Murine calvarial osteoblasts were transfected with miC, miR-487b-3p and anti-miR-487b-3p for $48 \mathrm{~h}$ and ALP activity in murine osteoblasts was measured. (B) ALP transcript levels were measured by $\mathrm{qPCR}$. (C) Murine osteoblasts stained with alizarin red-S. Representative photomicrographs show mineralized nodules in different groups. (D) Alizarin red-S Stain was extracted and quantified. (Bar $=400 \mu \mathrm{m})$. $(\mathrm{E})$ Change in miR-487b-3p expression during osteoblast differentiation. (F, G, H and I) qRT-PCR analysis of osteoblast marker genes type 1 collagen, OCN, Runx-2 BMP-2 normalized to GAPDH at $48 \mathrm{~h}$. All values represent means \pm s.E. $(n=6) . * P<0.05, * * P<0.01, * * * P<0.001$ compared with the control. A full colour version of this figure is available at https://doi.org/10.1530/ JOE-19-0015.
To recognize putative targets, in silico approach was carried out for genes in their 3-UTR (un-translated region) using open access software including mirdb and target scan. Among the numerous genes that were projected to be the possible targets by the above cited softwares, we concentrated on Nrarp which showed a 7-nt seed sequence match with miR-487b-3p in 3'-UTR region. The seed sequence in 3'-UTR region of Nrarp was highly conserved among mammals including mouse, squirrel, cow, cat, Rhesus monkey, humans and so forth (Fig. 3A). To confirm in silico data, osteoblast cells were transfected with $\mathrm{miC}$ or miR-487b-3p or anti-miR-487b-3p and mRNA levels of Nrarp were checked. The assay revealed that overexpression of miR-487b-3p downregulated Nrarp mRNA relative to the control (Fig. 3B) while transfection with antimiR-487b-3p reversed this effect. This result was supported by luciferase reporter assay. Luciferase reporter constructs containing the 3-UTR of Nrarp is depicted in Fig. 3C. The 3 '-UTR Nrarp clone or control clone was co-transfected with miC or miR-487b-3p in MCOs and level of luciferase enzyme action was calculated to decide the effects of miR-487b-3p on luciferase translation. The data suggested that overexpression of miR-487b-3p repressed the luciferase activity of the reporter gene in Nrarp 3-UTR-transfected cells (Fig. 3D). On the other hand, overexpression of miR-487b-3p had no seeming effect on luciferase activity 
A

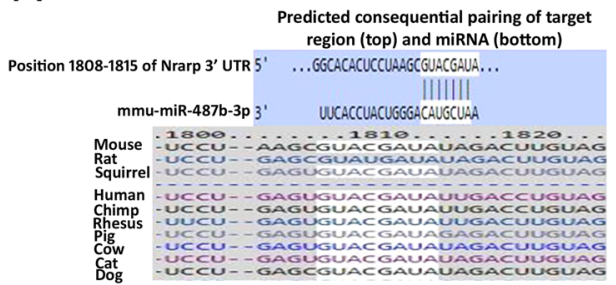

C

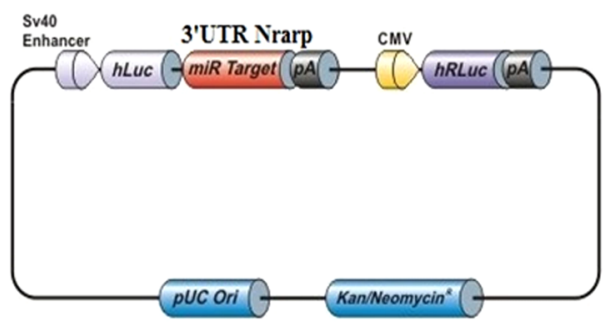

B
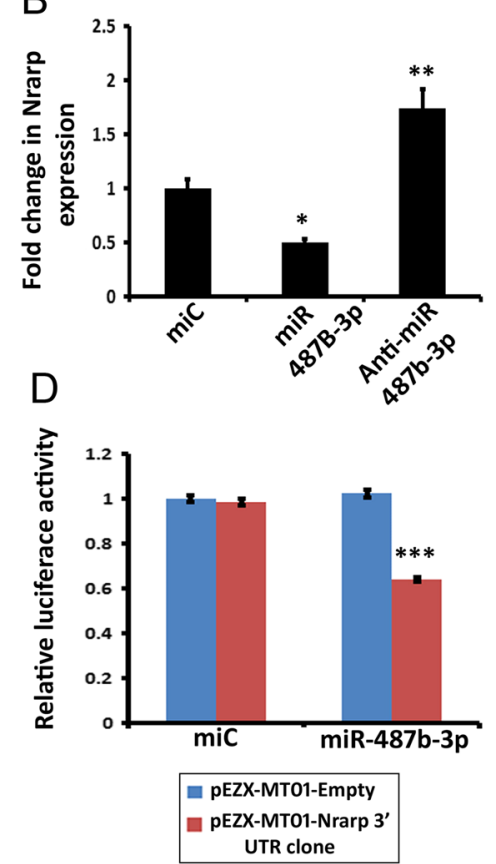

Figure 3

Identification of miR-487b-3p target genes in osteoblast differentiation. (A) Sequence alignment of Nrarp 3' UTR in various species and predicted consequential pairing of Nrarp 3' UTR and miR-487b-3p (B) Cells were transfected with the miC, miR-487b-3p and anti-miR-487b-3p and qRT-PCR analysis for Nrarp expression was performed. (C) Schematic presentation of the reporter plasmid used to illustrate the effect of Nrarp 3' UTR on luciferase activity. CMV, cytomegalovirus promoter; Luc, luciferase. (D) Effect of miR-487b-3p overexpression on a dual-luciferase reporter plasmid containing the Nrarp 3'UTR. All values represent means \pm S.E. $(n=6) .{ }^{*} P<0.05, * * P<0.01, * * * P<0.001$ compared with the control. A full colour version of this figure is available at https://doi.org/10.1530/ JOE-19-0015. in cells which were transfected with control vector authorizing the specificity of the target that is Nrarp gene (Fig. 3D).

\section{miR-487b-3p regulates canonical Wnt and Notch signaling by targeting Nrarp}

As mentioned earlier miR-487b-3p directly targets Nrarp, thus, our objective was to examine if miR-487b-3p (mimic) inhibits osteoblast functions by suppressing Wnt signaling and upregulating Notch signaling. To analyse whether miR-487b-3p was playing a role in regulating canonical Wnt and Notch signaling by modulating LEF1 and Notch protein, MCOs cells were transfected with $\mathrm{miC}$ (negative control), miR-487b-3p (mimic) and anti-miR-487b-3p (inhibitor) at $50 \mathrm{nM}$ concentration for western blot investigation. Cell lysate collected at $48 \mathrm{~h}$ after transfection revealed that transfection of calvarial osteoblast cells with miR-487b-3p (mimic) significantly reduced the protein levels of Nrarp, RUNX-2, Lef1 and $\beta$ catenin. Reverse observations were seen when cells were transfected with anti-miR-487b-3p. The protein levels of Notch1, Hes1 (a downstream Notch signaling target) and $\mathrm{P}-\beta$ catenin (component of Wnt signaling cascade) were upregulated when MCOs were transfected with miR-487b-3p and the outcomes were reversed when cells were transfected with anti-miR-487b-3p that is protein levels of Notch1, Hes1 and P- $\beta$ catenin were downregulated (Fig. 4A, B, C, D, E, F, G, H and I). Thus, these results undoubtedly show that miR-487b-3p regulates canonical Wnt and Notch signaling via targeting Nrarp.

\section{Silencing of miR-487b-3p in neonatal Balb/c mice eliminates the inhibitory effect of miR-487b-3p}

This study was carried out by subcutaneously injecting miR-487b-3p and anti-miR-487b-3p mixed with vivojetPEI reagent (Wahlquist et al. 2014) behind the head region in 1- to 2-day-old neonatal Balb/c mice. After 48-h injection, RNA was isolated from the calvariae of miR487b-3p and anti-miR-487b-3p treated neonatal Balb/c pups. It was detected that mRNA expression of osteogenic gene markers like Runx-2, type $1 \mathrm{Col}, \mathrm{OCN}, \mathrm{BMP}-2$ and $\beta$ catenin were significantly down in miR-487b-3p -treated mice. Opposite results were observed in anti-miR-487b-3ptreated mice (Fig. 5A, B, C, D, E, F and G). The expression of Hes1 (Notch signaling protein) was upregulated in miR-487b-3p, whereas opposite results were seen in antimiR-487b-3p-treated Balb/C pups. All these observations lead to the conclusion that miR-487b-3p negatively (adversely) regulates osteoblast differentiation.

\section{Anti-miR-487b-3p positively enhances in vivo bone formation}

To address the function of $\mathrm{miC}$ (negative control), miR-487b-3p (mimic) and anti-miR-487b-3p (inhibitor) in vivo, we carried out an experiment in four groups (sham, 


\begin{tabular}{l|l|l|l|l|}
$\begin{array}{l}\text { Journal of } \\
\text { Endocrinology }\end{array}$ & A A John et al. & $\begin{array}{l}\text { miR-487b-3p inhibits } \\
\text { osteoblast functions }\end{array}$ & $241: 3$ & 258 \\
\hline
\end{tabular}
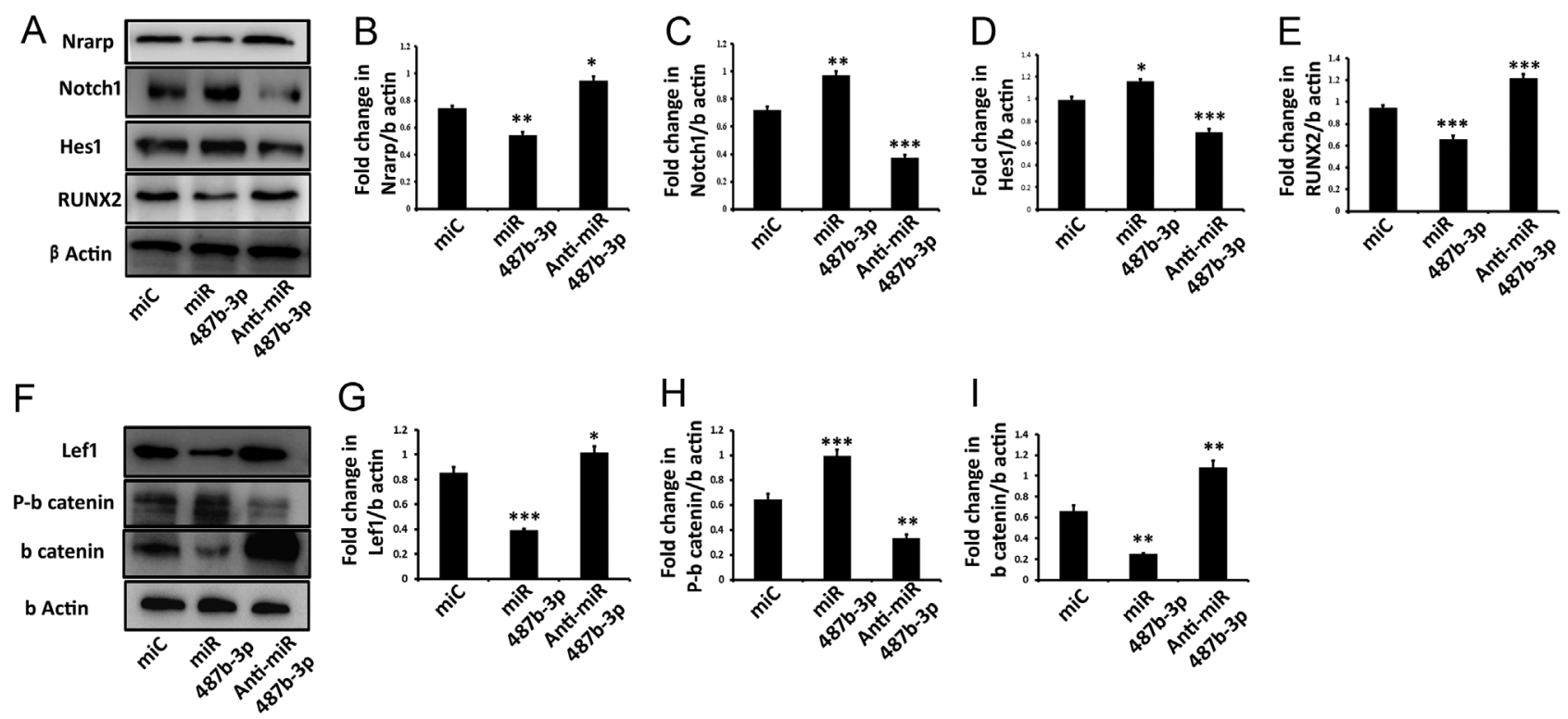

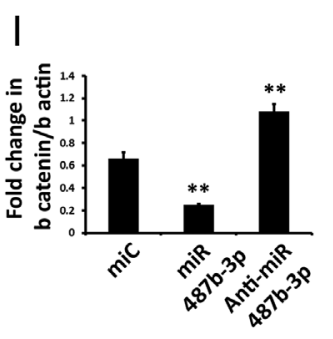

Figure 4

miR-487b-3p regulates canonical Wnt and Notch signaling by modulating LEF1 and Notch protein turnover via targeting Nrarp (A) Western blot analysis for Nrarp, Notch-1, Hes-1, RUNX-2 was performed after transfection with miC, miR-487b-3p and anti-miR-487b-3p. $\beta$-Actin was used as an internal control. (B, C, D and E) Densitometric analysis of the western blots. (F) Western blot analysis for Lef-1, P- $\beta$ catenin, $\beta$ catenin. (G, H and I) Densitometric analysis of the western blots. All values represent means \pm s.E. $(n=6)$. ${ }^{*} P<0.05$ and $* * * P<0.001$ compared with the control.

negative control, mimic and inhibitor) of female BALB/c mice weighing $25 \mathrm{~g}$. Three of the groups (negative control, mimic and inhibitor) underwent ovariectomy by the dorsal approach and the fourth group was sham operated. Ovariectomy leads to estrogen depletion (major cause of post menopausal osteoporosis) resulting in bone loss in trabeular component of long bones like femur and tibia. At the end of 1 month of ovariectomy, groups namely negative control, mimic and inhibitor were injected subcutaneously (one injection/week) for 3 weeks with $\mathrm{miC}$, miR-487b-3p and anti-miR-487b-3p, respectively, at a dose of $5 \mu \mathrm{g} /$ animal (each animal receives $15 \mu \mathrm{g}$ of oligonucleotide in 3 weeks). At the end of fourth week, the Balb/c mice were killed and femur bones were isolated and stored in $70 \%$ isopropanol for the investigation of trabecular microarchitecture via $\mu \mathrm{CT}$. Experimental strategy is given in (Fig. 6A). The 3D images undoubtedly disclose that miR-487b-3p has negative (adverse) effect on trabecular bone microarchitecture (Fig. 6B, C, D and E). Additional quantification of trabecular bone parameters i.e., BV/TV (bone volume/tissue volume ratio), Tb.N. (trabecular number), Tb.Th. (trabecular thickness), Tb.Sp. (trabecular separation), Tb.Pf. (trabecular pattern factor) and Conn.Dn. (connectivity density) were performed using $\mu \mathrm{CT}$. The $\mu \mathrm{CT}$ data publicized that anti-miR-487b-3ptreated group exhibited significant increase in $\mathrm{BV} / \mathrm{TV}$, Tb.N, Tb.Th and Conn Dn. with a simultaneous decrease
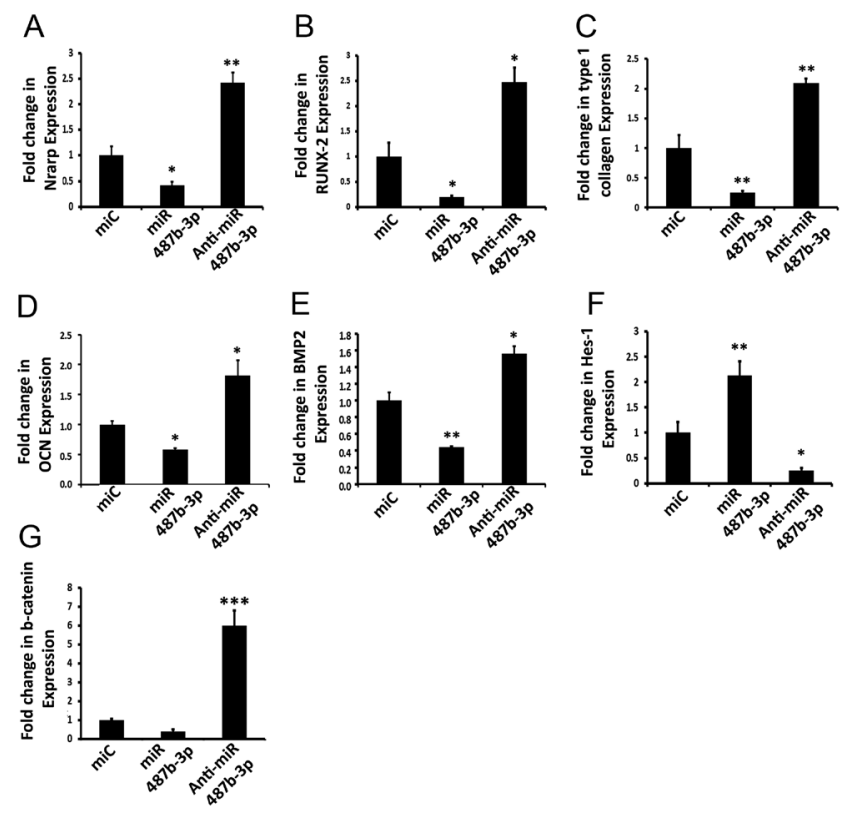

\section{Figure 5}

Silencing of miR-487b-3p abolished the inhibitory effect of miR-487b-3p on osteoblast differentiation. (A, B, C, D, E, F and G) Solutions of anti-miR-487b-3p, miR-487b-3p and miC were each diluted with in vivo-jetPEl solution and subcutaneously injected behind the head of neonatal mice. RNA isolated from calvariae to determine the transcript levels of Nrarp, RUNX-2, type I collagen, OCN, BMP-2, Hes-1 and $\beta$-catenin. All values represent means \pm S.E. $(n=6)$. ${ }^{*} P<0.05$, ** $P<0.01$ and $\star \star \star P<0.001$ compared with the control. 


\begin{tabular}{l|l|l|l|l|}
$\begin{array}{l}\text { Journal of } \\
\text { Endocrinology }\end{array}$ & A A John et al. & $\begin{array}{l}\text { miR-487b-3p inhibits } \\
\text { osteoblast functions }\end{array}$ & $241: 3$ & 259 \\
\hline
\end{tabular}
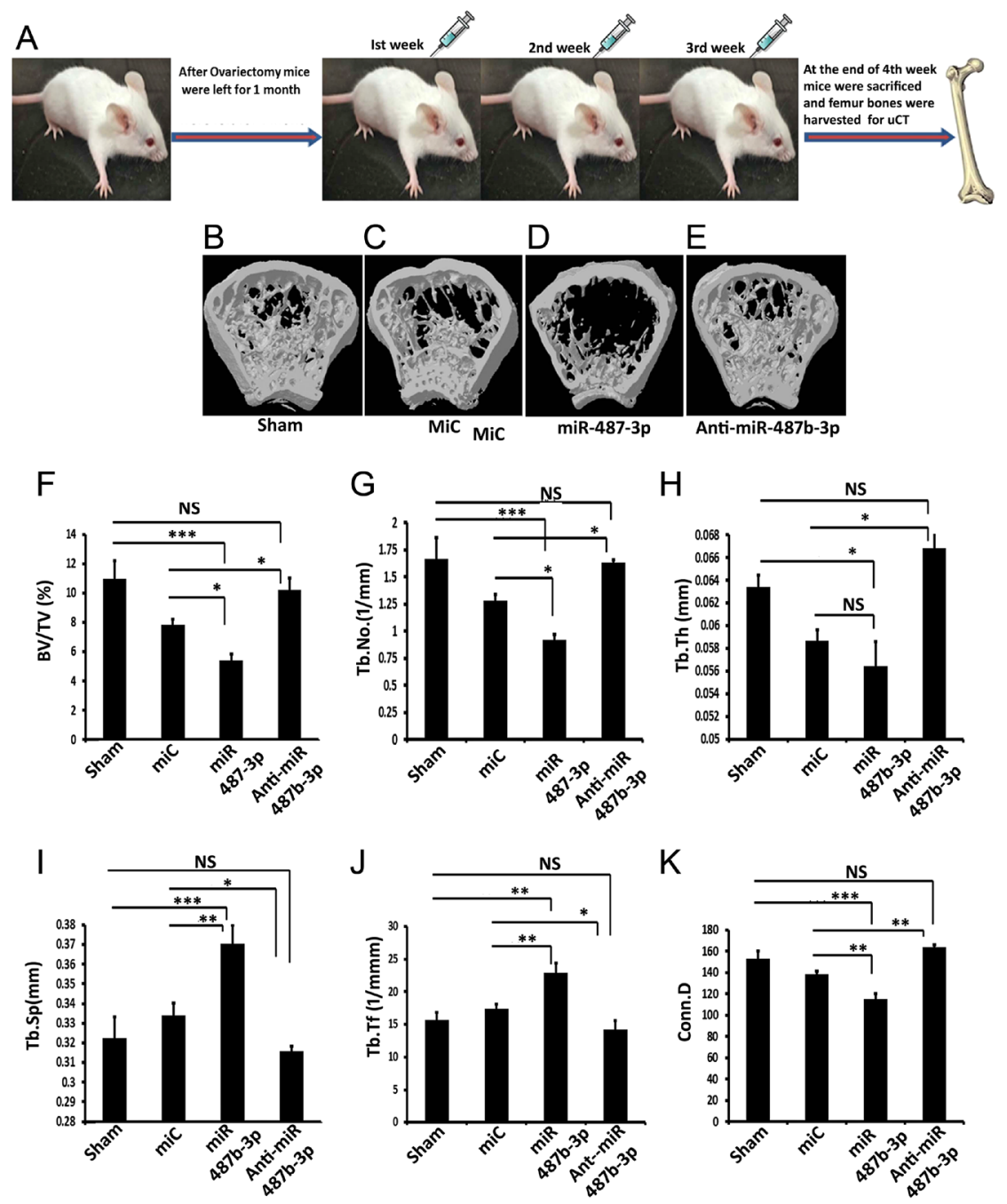

\section{Figure 6}

miR-487b-3p negatively regulates trabecular bone formation (A) Study plan of the study. (B, C, D and E) 3D micro-computed tomography of femur bone. $(F, G, H, I$, J and $K)$ Bone parameters, including BV/TV, Tb.N, Tb.Th, Conn.D, Tb.Sp and Tb.Pf in femora. All values represent means \pm s.E. $(n=6) .{ }^{*} P<0.05, * * P<0.01, * \star * P<0.001$ compared with the miC. BV/TV., bone volume/ tissue volume ratio; Conn. D., connectivity density; Tb.Sp., trabecular separation; Tb.N., trabecular number; Tb.Th., trabecular thickness; Tb.Pf., trabecular pattern factor. A full colour version of this figure is available at https://doi.org/10.1530/ JOE-19-0015.

in Tb. Sp and Tb. Pf in femora. All these parameters were reversed in miR-487b-3p treated group compared to miC (negative control) group (Fig. 6F, G, H, I, J and K). Various trabecular bone parameters between shamoperated group and anti-miR-487b-3p-treated group were almost equal. Various bone strength parameters (power, energy and stiffness) were significantly increased in antimiR-487b-3p-treated group, while this effect was reversed in miR-487b-3p-treated group compared to miC that is negative control group (Fig. 7A, B and C). Importantly, it was observed that mice treated with miR-487b-3p showed significant dip in serum levels of P1NP (bone formation marker), while serum P1NP levels were equal to that of sham in anti-miR-487b-3p-treated mice (Fig. 7D). CTx (bone resorption marker) levels, on the other hand, were greater in miR-487b-3p-treated mice, while CTx levels were brought back to sham levels in anti-miR-487b-3p-treated mice (Fig. 7E). Further histological data (H\&E staining) revealed that there is scarce and thinning trabecula, with loss of connectivity in miR-487b-3p-treated femur bones, while anti-miR-487b-3p treatment significantly protected trabecular bone and showed greater connectivity (Fig. 7F, G, H, I, J, K, L and M).

\section{Discussion}

This study has recognized an unknown function of miR-487b-3p in bone where it suppresses osteogenesis by repressing Nrarp. Previous studies report that overexpression of miR-487b-3p mimics the effects of GRM3 (metabotropic glutamate receptor 3) knockdown and suppresses the tumorigenicity of colon cancer cells in vivo. It has been discovered that GRM3 is expressed in mammalian central nervous system and is significantly upregulated in majority of human colonic adenocarcinomas tested and colon cancer cell lines (Yi et al. 2017). miR-487b-3p was chosen as it was more 

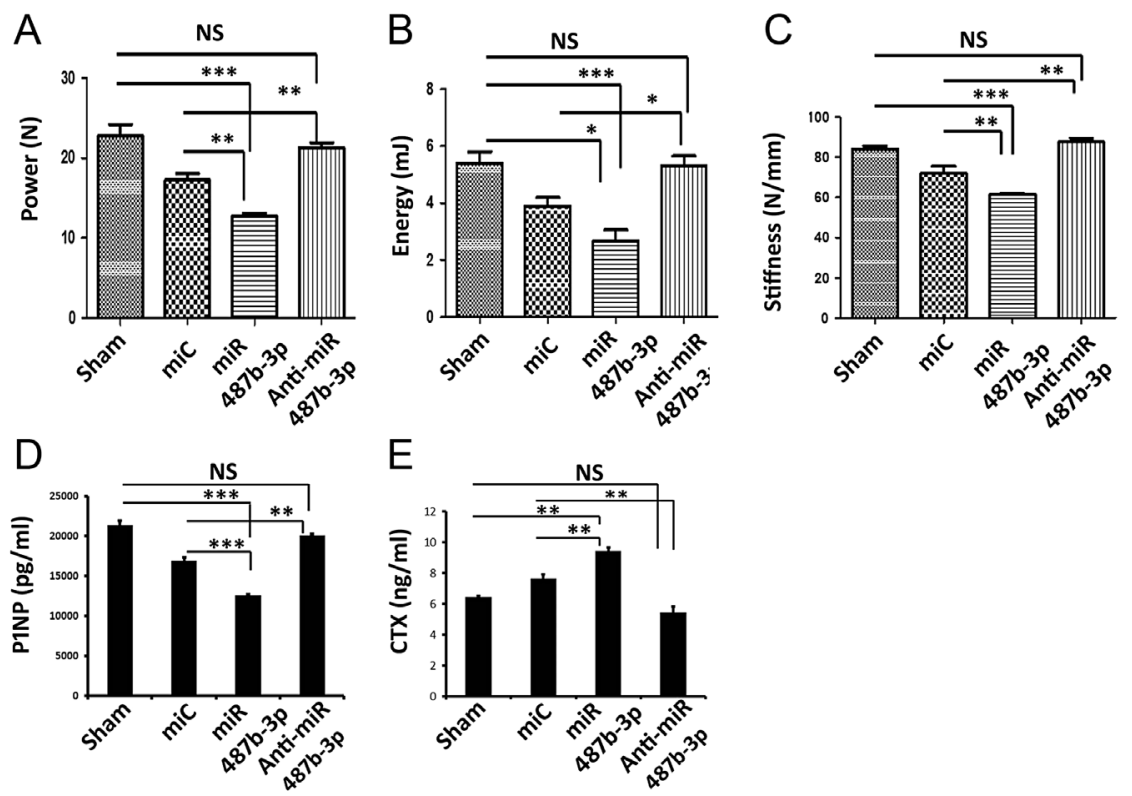

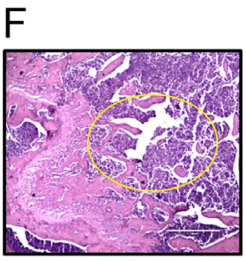

Sham (10)

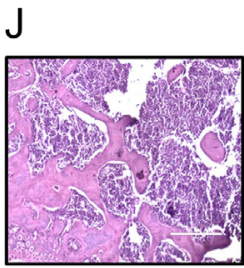

Sham (20X)

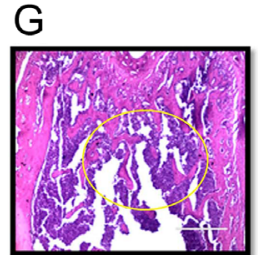

$\operatorname{miC}(10 x)$

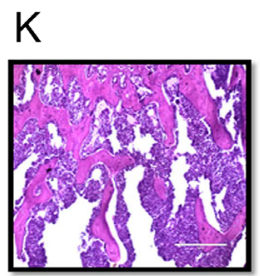

$\operatorname{miC}(20 x)$

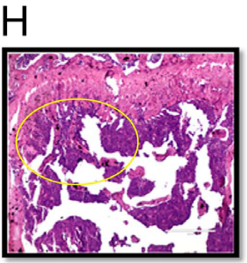

miR-487b-3p (10X)

$\mathrm{L}$

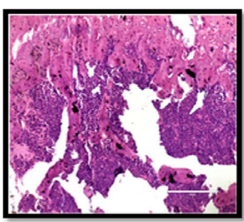

miR-487b-3p (20X)

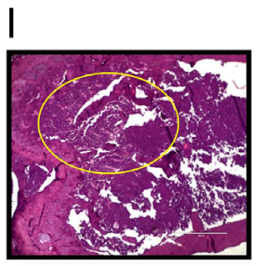

Anti-miR-487b-3p (10X)

$\mathrm{M}$

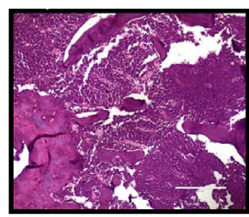

Anti-miR-487-3p (20X)

\section{Figure 7}

Effect of miR-487b-3p and anti-miR-487b-3p on bio-mechanical properties of isolated femur. (A) Power, (B) energy, (C) stiffness. (D) Serum levels of amino-terminal propeptide of type 1 procollagen (serum P1NP) (E) C-terminal telopeptide of type I collagen (serum CTx). (F) Histological analysis by $\mathrm{H} \& \mathrm{E}$ staining in ( $\mathrm{F}$ and J) Sham at $10 \times$ and $20 \times(\mathrm{G}$ and $\mathrm{K}) \mathrm{miC}$ at $10 \times$ and $20 \times(\mathrm{H}$ and $\mathrm{L}) \mathrm{miR}-487 \mathrm{~b}-3 \mathrm{p}$ at $10 \times$ and $20 \times(\mathrm{I}$ and $\mathrm{M})$ anti-miR-487b-3p at $10 \times$ and $20 \times$. than 6.5-fold downregulated in MCO treated with medicarpin. The mechanism of action in regulation of osteoblast functions is unknown. To corroborate the results of microRNA profiling, expression of miR-487b-3p in medicarpin and bone morphogenetic protein 2 (BMP-2) treated osteoblast cells was determined. It was witnessed that incitement of osteoblast differentiation with medicarpin or BMP-2 led to significant reduction in miR-487b-3p transcript levels. These data were strengthened by the fact that Balb/c mice treated with medicarpin for 1 month also exhibited reduced transcript levels of miR-487-3p in long bones.

To decide if miR-487b-3p specifically controls osteoblast differentiation, we examined the activity of miR-487b-3p during osteoblastogenesis. MicroRNA-487b-3p overexpression repressed osteoblast differentiation, mineralization and transcript and protein levels of many osteogenic markers like Runx-2, BMP-2, Type I col and OCN. Reverse outcomes were seen in anti-miR-487-3ptreated MCOs. These results were validated in human osteoblasts where overexpression of miR-487-3p prompted diminished ALP action and decreased mRNA levels of osteogenic gene markers.

To investigate the mechanism by which miR-487b-3p directs osteoblastogenesis, we explored for potential target genes that have a well-known role in enhancing osteogenesis. These target genes were identified using target prediction tools like target scan and mirdb. It was revealed that miR-487b-3p targets several genes; however, one of the thought-provoking target for us was Nrarp. Nrarp is a small protein with two ankyrin repeats (Krebs et al. 2001). It is highly conserved in higher vertebrates (Krebs et al. 2001). Overexpression of Nrarp in Xenopus embryo inhibited Notch signaling (Lamar et al. 2001). 
The in silico observation was validated by decreased Nrarp transcript levels in miR-487b-3p-transfected cells, while upregulated expression of Nrarp was found in antimiR-487b-3p-transfected calvarial osteoblasts. This result was supported by luciferase reporter assay where it was found that overexpression of mimic miR-487b-3p suppressed the luciferase activity of the Nrarp 3'-UTR reporter construct.

It is important to note that Nrarp acts as a component of a negative feedback loop to destabilize NICD (Krebs et al. 2001, Borggrefe et al. 2016), a constitutively active form of Notch-1, thus downregulating Notch signals including Hes and Hey (Borggrefe et al. 2016). Notch-1 signaling controls osteoblast differentiation by suppressing Runx-2 (Ann et al. 2011). Thus, Nrarp-mediated destabilization of NICD releases the inhibitory effect of Notch signaling on Runx-2 thereby promoting osteoblast differentiation. Established on these observations we hypothesized that miR-487b-3p (mimic) might be obstructing osteoblast functions by targeting Nrarp-mediated inhibition of Notch signaling. Our hypothesis was supported by increased protein levels of Notch-1 and Hes1 and reduced protein levels of Nrarp and Runx-2 in miR-487b-3p-transfected cells. It was interesting to observe that these outcomes were reversed in cells transfected with anti- miR-487b-3p.

Nrarp also acts as a positive regulator of Wnt signaling pathway where it stabilizes LEF-1, an important transcription factor in Wnt signaling cascade thereby obstructing its ubiquitination (Ishitani et al. 2005, Hoeppner et al. 2009). Reduced protein levels of LEF-1 and beta catenin were found in miR-487b-3p-treated MCOs, while opposite effects were observed in anti-miR-treated MCOs. Thus, miR-487b-3p exerts its inhibitory role in osteoblast differentiation by targeting Nrarp thereby promoting the suppression of Runx-2 and Wnt signaling.

As all these were in vitro studies carried out in osteoblast cells, we decided to silence miR-487b-3p in an ex vivo and in vivo animal model. In the ex vivo study expression of osteogenic gene markers and proteins like Nrarp, Hes-1 and $\beta$-catenin was determined in calvarial protein lysates. It was witnessed that in anti-miR-487b-3p (inhibitor)treated mice, expression of osteogenic markers was significantly higher as a result of reduced endogenous levels of miR-487-3p (mimic). Anti-miR-487b-3p transfection also led to increased expression of Nrarp and $\beta$-catenin. Thus, suppressed endogenous levels of miR-487b-3p stimulated Nrarp-mediated Notch signaling inhibition and stabilization of Wnt signaling pathway thereby promoting osteogenesis. In the in vivo study, miR-487b-3p mimic and its inhibitor was injected subcutaneously to Ovx BALB/c mice. Ovx mice model was used to carry out an in vivo study as it rightly imitates the important clinical feature of the estrogen-depleted human skeleton. Following ovariectomy, there is a decrease in trabeculae density and deterioration of bone microarchitecture. Our results show that inhibition of miR-487b-3p led to significant enhancement in trabecular microarchitecture in Ovx animals. On the other hand contrasting results were obtained in mimic i.e. miR-487b-3p-treated groups. These data suggest that miR-487b-3p negatively regulates bone formation. Anti-miR-treated groups also presented with better bone strength parameters and increased levels of bone formation marker, P1NP which were compromised in Ovx mimic-treated mice. Most importantly, silencing of miR-487b-3p led to a decrease in bone resorption marker CTx which were otherwise increased in mimic-treated groups.

Overall, these results reveal that targeting miR-487b-3p may be an interesting approach to study further its role in promoting new bone formation. Nevertheless, additional studies are required to confirm these observations as there are some limitations to our study. A single miRNA like miR-487b-3p has more than hundreds of predicted targets. It is not known if such target genes could participate in osteoblast functions mediated by miR-487b-3p. Moreover, as miRNAs regulate many genes, there is a risk of potential off target effects. For instance, miR-487b-3p regulates colon adenocarcinoma and its inhibition may have the opportunity of some bad effects in disorders of colon cancers.

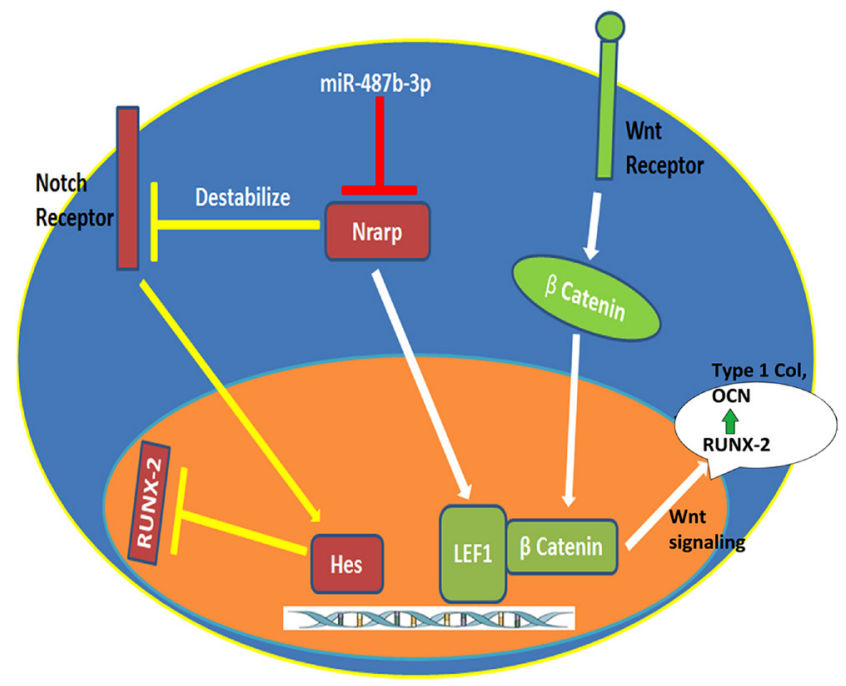

\section{Figure 8}

A proposed model for miR-487b-3p mediated suppression of osteoblast functions. miR-487b-3p functions as a negative regulator of osteogenesis by repressing Nrarp expression, which in turn, suppresses Runx-2 and destabilizes LEF-1, both of which play a pivotal role in osteoblast differentiation. A full colour version of this figure is available at https:// doi.org/10.1530/JOE-19-0015. 
Additionally, our results though are suggestive of miR-487b-3p being targeted to bone cells, but to ascertain this observation, it will be requisite to deliver $\mathrm{miR}$ inhibitors precisely into the target cells. Thus, need for ideal delivery systems like Nano carriers or high-density lipoproteins nanoparticles cannot be undermined.

In conclusion, our results suggest that miR-487b-3p functions as a negative regulator of osteogenesis by blocking Nrarp expression, which in turn, suppresses Runx-2 and Wnt signaling, both of which play a pivotal role in osteoblast differentiation (Fig. 8). As miR-487-3p targets Nrarp, an inhibitor of Notch signaling which plays an important role in skeletal development and bone remodeling, it will be interesting to study role of miR-487-3p during bone development.

\section{Supplementary data}

This is linked to the online version of the paper at https://doi.org/10.1530/ JOE-19-0015.

\section{Declaration of interest}

The authors declare that there is no conflict of interest that could be perceived as prejudicing the impartiality of the research reported.

\section{Funding}

Department of Biotechnology (DBT), Government of India and Centre for Research in Anabolic Skeletal Targets in Health and Illness (ASTHI), Council of Scientific and Industrial Research, Government of India.

\section{Author contribution statement}

Experiments were conceived and designed by D S and A AJ. A A J performed the experiments and analyzed the data. D S wrote the paper. R P assisted in the experiments and helped in the analysis of the data. All authors have read and approved the manuscript before submission.

\section{Acknowledgements}

Fellowship grants from the Council of Scientific and Industrial Research (A A J), University Grants Commission (R P), Government of India are acknowledged. The CDRI communication number is 9836 .

\section{References}

Ann EJ, Kim HY, Choi YH, Kim MY, Mo JS, Jung J, Yoon JH, Kim SM, Moon JS, Seo MS, et al. 2011 Inhibition of Notch1 signaling by Runx2 during osteoblast differentiation. Journal of Bone and Mineral Research 26 317-330. (https://doi.org/10.1002/jbmr.227)
Bae Y, Yang T, Zeng HC, Campeau PM, Chen Y, Bertin T, Dawson BC, Munivez E, Tao J \& Lee BH 2012 miRNA-34c regulates Notch signaling during bone development. Human Molecular Genetics 21 2991-3000. (https://doi.org/10.1093/hmg/dds129)

Borggrefe T, Lauth M, Zwijsen A, Huylebroeck D, Oswald F \& Giaimo BD 2016 The Notch intracellular domain integrates signals from Wnt, Hedgehog, TGFbeta/BMP and hypoxia pathways. Biochimica and Biophysica Acta 1863 303-313. (https://doi.org/10.1016/j. bbamcr.2015.11.020)

Chen Q, Shou P, Zheng C, Jiang M, Cao G, Yang Q, Cao J, Xie N, Velletri T, Zhang X, et al. 2016 Fate decision of mesenchymal stem cells: adipocytes or osteoblasts? Cell Death and Differentiation 23 1128-1139. (https://doi.org/10.1038/cdd.2015.168)

Deregowski V, Gazzerro E, Priest L, Rydziel S \& Canalis E 2006 Notch 1 overexpression inhibits osteoblastogenesis by suppressing Wnt/ beta-catenin but not bone morphogenetic protein signaling. Journal of Biological Chemistry 281 6203-6210. (https://doi.org/10.1074/jbc. M508370200)

Dontu G, Jackson KW, McNicholas E, Kawamura MJ, Abdallah WM \& Wicha MS 2004 Role of Notch signaling in cell-fate determination of human mammary stem/progenitor cells. Breast Cancer Research 6 R605-R615. (https://doi.org/10.1186/bcr920)

Furukawa T, Ishifune C, Tsukumo SI, Hozumi K, Maekawa Y, Matsui N, Kaji R \& Yasutomo K 2016 Transmission of survival signals through Delta-like 1 on activated CD4(+) T cells. Scientific Reports 633692. (https://doi.org/10.1038/srep33692)

Grimson A, Farh KK, Johnston WK, Garrett-Engele P, Lim LP \& Bartel DP 2007 MicroRNA targeting specificity in mammals: determinants beyond seed pairing. Molecular Cell 27 91-105. (https://doi. org/10.1016/j.molcel.2007.06.017)

Hoeppner LH, Secreto F, Jensen ED, Li X, Kahler RA \& Westendorf JJ 2009 Runx 2 and bone morphogenic protein 2 regulate the expression of an alternative Lef1 transcript during osteoblast maturation. Journal of Cellular Physiology 221 480-489. (https://doi.org/10.1002/jcp.21879)

Ishitani T, Matsumoto K, Chitnis AB \& Itoh M 2005 Nrarp functions to modulate neural-crest-cell differentiation by regulating LEF1 protein stability. Nature Cell Biology 7 1106-1112. (https://doi.org/10.1038/ ncb1311)

Jensen ED, Gopalakrishnan R \& Westendorf JJ 2010 Regulation of gene expression in osteoblasts. BioFactors 36 25-32. (https://doi. org/10.1002/biof.72)

John AA, Prakash R, Kureel J \& Singh D 2018 Identification of novel microRNA inhibiting actin cytoskeletal rearrangement thereby suppressing osteoblast differentiation. Journal of Molecular Medicine 96 427-444. (https://doi.org/10.1007/s00109-018-1624-y)

John B, Enright AJ, Aravin A, Tuschl T, Sander C \& Marks DS 2004 Human microRNA targets. PLoS Biology 2 e363. (https://doi. org/10.1371/journal.pbio.0020363)

Krebs LT, Deftos ML, Bevan MJ \& Gridley T 2001 The Nrarp gene encodes an ankyrin-repeat protein that is transcriptionally regulated by the notch signaling pathway. Developmental Biology 238 110-119. (https://doi.org/10.1006/dbio.2001.0408)

Kureel J, Dixit M, Tyagi AM, Mansoori MN, Srivastava K, Raghuvanshi A, Maurya R, Trivedi R, Goel A \& Singh D 2014 miR-542-3p suppresses osteoblast cell proliferation and differentiation, targets BMP-7 signaling and inhibits bone formation. Cell Death and Disease $\mathbf{5}$ e1050. (https://doi.org/10.1038/cddis.2014.4)

Kureel J, John AA, Dixit M \& Singh D 2017 MicroRNA-467g inhibits new bone regeneration by targeting Ihh/Runx-2 signaling. International Journal of Biochemistry and Cell Biology 85 35-43. (https://doi. org/10.1016/j.biocel.2017.01.018)

Kureel J, John AA, Prakash R \& Singh D 2018 miR 376c inhibits osteoblastogenesis by targeting Wnt3 and ARF-GEF-1-facilitated augmentation of beta-catenin transactivation. Journal of Cellular Biochemistry 119 3293-3303. (https://doi.org/10.1002/jcb.26490) https://joe.bioscientifica.com https://doi.org/10.1530/JOE-19-0015 (c) 2019 Society for Endocrinology Published by Bioscientifica Ltd. Printed in Great Britain 
Lai EC, Tam B \& Rubin GM 2005 Pervasive regulation of Drosophila Notch target genes by GY-box-, Brd-box-, and K-box-class microRNAs. Genes and Development 19 1067-1080. (https://doi.org/10.1101/gad.1291905)

Lamar E, Deblandre G, Wettstein D, Gawantka V, Pollet N, Niehrs C \& Kintner C 2001 Nrarp is a novel intracellular component of the Notch signaling pathway. Genes and Development 15 1885-1899. (https://doi. org/10.1101/gad.908101)

Poulton JS, Huang YC, Smith L, Sun J, Leake N, Schleede J, Stevens LM \& Deng WM 2011 The microRNA pathway regulates the temporal pattern of Notch signaling in Drosophila follicle cells. Development 138 1737-1745. (https://doi.org/10.1242/dev.059352)

Regan J \& Long F 2013 Notch signaling and bone remodeling. Current Osteoporosis Reports 11 126-129. (https://doi.org/10.1007/s11914-0130145-4)

Wahlquist C, Jeong D, Rojas-Munoz A, Kho C, Lee A, Mitsuyama S, van Mil A, Park WJ, Sluijter JP, Doevendans PA, et al. 2014 Inhibition of miR-25 improves cardiac contractility in the failing heart. Nature $\mathbf{5 0 8}$ 531-535. (https://doi.org/10.1038/nature13073)
Won KY, Kim YW, Kim HS, Lee SK, Jung WW \& Park YK 2013 MicroRNA$199 \mathrm{~b}-5 \mathrm{p}$ is involved in the Notch signaling pathway in osteosarcoma. Human Pathology 44 1648-1655. (https://doi.org/10.1016/j. humpath.2013.01.016)

Yavropoulou MP \& Yovos JG 2014 The role of Notch signaling in bone development and disease. Hormones 13 24-37. (https://doi. org/10.1007/BF03401318)

Yi H, Geng L, Black A, Talmon G, Berim L \& Wang J 2017 The miR487b-3p/GRM3/TGFbeta signaling axis is an important regulator of colon cancer tumorigenesis. Oncogene 36 3477-3489. (https://doi. org/10.1038/onc.2016.499)

Zanotti S \& Canalis E 2016 Notch signaling and the skeleton. Endocrine Reviews 37 223-253. (https://doi.org/10.1210/er. 2016-1002)

Zanotti S, Smerdel-Ramoya A, Stadmeyer L, Durant D, Radtke F \& Canalis E 2008 Notch inhibits osteoblast differentiation and causes osteopenia. Endocrinology 149 3890-3899. (https://doi.org/10.1210/ en.2008-0140)

Received in final form 8 April 2019

Accepted 12 April 2019

Accepted Preprint published online 12 April 2019 (c) 2019 Society for Endocrinology Published by Bioscientifica Ltd.
Printed in Great Britain 